\title{
Approximate Reasoning through Multigranular Approximate Rough Equalities
}

\author{
B. K. Tripathy \\ School of Computer Science and Engineering, VIT University, Vellore-632014, Tamilnadu, India \\ Email: tripathybk@vit.ac.in \\ Rashmi Rawat \\ School of Computer Science and Engineering, VIT University, Vellore-632014, Tamilnadu, India \\ Email: rashmirawattt@gmail.com \\ Divya Vani .Y \\ School of Computer Science and Engineering, VIT University, Vellore-632014, Tamilnadu, India \\ Email: divyasudha99@gmail.com \\ Sudam Charan Parida \\ Lecturer in Mathematics, K.B.V. Mahavidyalaya, Kabisurya Nagar, Ganjam, Odisha- 761104 \\ Email: sudamparida32@gmail.com
}

\begin{abstract}
The notion of rough set was introduced by Pawlak as an uncertainty based model, which basically depends upon single equivalence relations defined over a universe or a set of equivalence relations, which are not considered simultaneously. Hence, from the granular computing point of view it is unigranular by nature. Qian et al in 2006 and in 2010 introduced two types of multigranular rough sets (MGRS) called the optimistic and pessimistic MGRS respectively. The stringent notion of mathematical equality of sets was extended by introducing a kind of approximate equality, called rough equality by Novotny and Pawlak, which uses basic rough sets. Later three more related types of such approximate equalities have been introduced by Tripathy et al. He has also provided a comparative analysis of these four types of approximate equalities of sets leading to approximate reasoning in real life situations. Two of these four types of approximate equalities; namely the rough equality and rough equivalence have been extended to the context of multigranulations by Tripathy et al very recently. In this paper we carry out this study further by introducing the notion of approximate rough equalities for multigranulations and establish their properties. We use a real life example to illustrate the results in the paper and also to construct examples in support of some parts of the properties.
\end{abstract}

Index Terms - Rough Set, Approximate Equalities, Optimistic Multigranulations, Pessimistic Multigranulations, Bottom REqual, Top R-Equal, R-Equal, Approximate R-Equal

\section{INTRODUCTION}

In modern days vagueness has become a common feature in databases. Several approaches have been made to study such type of databases. Notable among them are the notion of fuzzy sets introduced by Zadeh [1] and the notion of rough sets introduced by Pawlak [2]. Since its induction Rough set based techniques have been found to be useful in handling uncertainty in databases, database anonymization, ru le generation and reduction of attributes
[3]. The basic rough set theory depends on the notion of equivalence relations defined over a universe. But it uses a single equivalence relation at a time either directly or indirectly. So, it is unigranular from the granular computing point of $\mathrm{v}$ iew. Extending the idea of considering a single equivalence relation for granularity of knowledge, the notions of multigranulations were introduced by Qian et al [4, 5] recently. In fact they introduced the concepts of optimistic and pessimistic multigranular rough sets (MGRS). Several papers have been published since then. Some of the notable ones are the study of algebraic properties of MGRS [6, 7, 8], extensions of MGRS in incomplete information systems [6] and topological properties of MGRS [6]. Many real life examples have been provided in these papers.

The notion of equality of two sets used in mathematics is too stringent as they are said to be equal when they have same elements and are not that useful in most of the real life situations. In real life situations a user utilizes available knowledge in order to decide the equality of sets, where as there is no such scope in the case of mathe matical equality. Hence, Novotny and Pawlak [3, 9, $10,11]$ introduced a notion of approximate equality using rough sets, described in terms of two subtypes called lower rough equality and upper rough equality. The rough equality of sets incorporates human knowledge in deciding the equality of sets instead of comparing their elements only. It was observed in 2008 by Tripathy et al [12] that even this definition of rough equality boils down to equality of sets which is clear from the definition. So, they introduced the notion of rough equivalence, which is free from such deficiencies. Later on Tripathy [13] combined the conditions of rough equality and rough equivalence to define two more types of approximate equalities; called the approximate rough equality and approximate rough equivalence. In a sense, this 
completes the group of approximate equalities using the concepts of lower and upper approximations of sets. Also, a nice comparative analysis of relative strengths and weaknesses of these four types of approximate equalities has been made in [13] and it is established that the notion of approximate rough equivalence is the most general among the four.

Recently, two of the four types of rough equalities; namely the rough equality and rough equivalence, have been extended to the context of multigranulations by Tripathy et al $[12,14]$. It is natural to try to extend the other types to the context of mu ltigranulation and it is our aim to handle the concept of approximate rough equalities to the context of multigranulations and study their properties. In this paper, we deal with both general and replacement properties in terms of both types of multigranulations i.e. optimistic and pessimistic multigranulations.

The overall structure of the paper is as follows. In section 2 we provide definitions and notations to be used in the paper. In section 3 we present the multigranular rough equalities. In Section 4 we introduce the concepts of multigranular approximate equalities and establish their properties. We provide the conclusion in section 5 followed by the referred papers and other materials in section 6.

\section{DEFINITIONS AND NOT ATIONS}

In this section we provide some of the definitions and notations to be used. We start with the basic rough sets in the next sub section.

\section{A. Basic rough sets}

Let $U$ be a universe of discourse and $R$ be an equivalence relation over $U$. By $U / R$ we denote the family of all equivalence class of $R$, referred to as categories or concepts of $R$ and the equivalence class of an element $\mathrm{x} \in \mathrm{U}$, is denoted by $[\mathrm{x}]_{R}$. By a knowledge base, we understand a relational system $K=(U, \mathrm{Q})$, where $U$ is as above and $\mathrm{Q}$ is a family of equivalence relations over $U$. For any subset $\mathrm{P}(\neq \phi) \subseteq \mathrm{Q}$, the intersection of all equivalence relations in $\mathrm{P}$ is denoted by IND (P) and is called the indiscernibility relation over P. Given any $X \subseteq U$ and $R \in \mathrm{IND}(\mathrm{K})$, we (45) associate two subsets, $\quad \underline{R} X=\bigcup\{Y \in U / R: Y \subseteq X\} \quad$ and $\bar{R} X=\bigcup\{Y \in U / R \mid Y \cap X \neq \phi\}$, called the R-lower and R-upper approximations of $X$ respectively.

The R-boundary of $X$ is denoted by $B N_{R}(X)$ and is given by $B N_{R}(X)=\bar{R} X-\underline{R} X$. The elements of $\underline{R} X$ are those elements of $U$, which can certainly be classified as elements of $\mathrm{X}$, and the elements of $\bar{R} X$ are those elements of $U$, which can possibly be classified as ele ments of $\mathrm{X}$, emp loying knowledge of $R$. We say that $X$ is rough with respect to $R$ if and only if $\underline{R} X \neq \bar{R} X$, equivalently $B N_{R}(X) \neq \phi . X$ is said to be R-definable if and only if $\bar{R} Y$, or $B N_{R}(X)=\phi$.

\section{B. Multigranular rough sets}

The concept of granular computing was introduced by Zadeh. According to this concept an equivalence relation on the universe can be regarded as a granulation, and a partition on the universe can be regarded as a granulation space. As mentioned earlier, from the granular computing point of view, two types of Multigranulations have been defined using rough sets.

The optimistic multigranular rough sets were introduced by Qian et al [4] as follows. We note that in the beginning there was only one type of Multigranulation and it was not named as optimistic. After the development of a second type of Multigranulation, the first one was called optimistic and the second one was called as pessimistic [5]. We note that we are considering two-granulations only. For granulations of higher order, the definitions and properties are similar. The notations used for the two types of Multigranulations were different in the original papers. But we follow the notations used in a recent paper by Tripathy et al $[6,7,8]$. That is we use $\mathrm{R}+\mathrm{S}$ for optimistic Multigranulation and $R * S$ for pessimistic Multigranulation, where $\mathrm{R}$ and $\mathrm{S}$ are two equivalence relations on $\mathrm{U}$.

Definition 1: Let $\mathrm{K}=(\mathrm{U}, \mathbf{R})$ be a knowledge base, $\mathbf{R}$ be a family of equivalence relations, $X \subseteq U$ and $R, S \in R$. We define [6] the optimistic multi-granular lower approximation and optimistic multi-granular upper approximation of $\mathrm{X}$ with respect to $\mathrm{R}$ and $\mathrm{S}$ in $\mathrm{U}$ as

$$
\begin{aligned}
& \underline{R+S} X=\left\{x \mid[x]_{R} \subseteq X \text { or }[x]_{S} \subseteq X\right\} \\
& \overline{R+S} X=\sim(\underline{R+S}(\sim X))
\end{aligned}
$$

Definition 2: Let $\mathrm{K}=(\mathrm{U}, \mathbf{R})$ be a knowledge base, $\mathbf{R}$ be a family of equivalence relations, $X \subseteq U$ and $R, S \in R$. We define [5] the pessimistic multi-granular lower approximation and pessimistic multi-granular upper approximation of $\mathrm{X}$ with respect to $\mathrm{R}$ and $\mathrm{S}$ in $\mathrm{U}$ as

$$
\begin{aligned}
& \underline{R * S} X=\left\{x \mid[x]_{R} \subseteq X \text { and }[x]_{S} \subseteq X\right\} \\
& \overline{R * S} X=\sim(\underline{R * S}(\sim X))
\end{aligned}
$$

Next we present some properties of multigranular rough sets, which shall be used in the proofs of the results of this paper $[7,8]$.

$$
\begin{aligned}
& \underline{R+S}(X \cap Y) \subseteq \underline{R+S}(X) \cup \underline{R+S}(Y) \\
& \underline{R+S}(X \cup Y) \supseteq \underline{R+S}(X) \cup \underline{R+S}(Y) \\
& \overline{R+S}(X \cap Y) \subseteq \overline{R+S}(X) \cap \overline{R+S}(Y) \\
& \overline{R+S}(X \bigcup Y) \supseteq \overline{R+S}(X) \cup \overline{R+S}(Y)
\end{aligned}
$$




$$
\begin{aligned}
& \underline{R^{*} S}(X \cup Y) \supseteq \underline{R^{*} \mathrm{~S}}(X) \cup \underline{R^{*} \mathrm{~S}}(Y) \\
& \overline{R^{*} \mathrm{~S}}(X \cap Y) \supseteq \overline{R^{*} \mathrm{~S}}(X) \cap \overline{R^{*} \mathrm{~S}}(Y) \\
& \underline{R^{*} S}(X \cap Y) \supseteq \underline{R^{*} \mathrm{~S}}(X) \cap \underline{R^{*} \mathrm{~S}}(Y) \\
& \overline{R^{*} \mathrm{~S}}(X \bigcup Y)=\overline{R^{*} \mathrm{~S}}(X) \cup \overline{R^{*} \mathrm{~S}}(Y)
\end{aligned}
$$

\section{Approximate equalities of rough sets}

As described in the introduction, sometimes exact equality (equality in the mathematical sense) is too stringent to apply in day to day life. We often talk about equality of sets or domains, which can be considered to be equal for the purpose or under the circumstances in real life situations. So, approximate equalities play a significant role in human reasoning. Also, it is dependent upon the knowledge the assessors have about the domain under consideration as a whole but mostly not the knowledge about individuals.

\section{Rough Equalities}

As a step to incorporate user knowledge in considering equality of sets, Novotny and Pawlak [2, 9, 10, 11] introduced the following concepts of rough equalities.

Let $\mathrm{K}=(\mathrm{U}, \mathbf{R})$ be a knowledge base, $X \subseteq U$ and $R \in I N D(K)$.

Definition 3: We say that

$\mathrm{X}$ and $\mathrm{Y}$ are bottom rough equal (X b_R_eq $\mathrm{Y}$ )

if and only if $\underline{R} X=\underline{R} Y$.

$\mathrm{X}$ and $\mathrm{Y}$ are top rough equal (X t_R_eq $\mathrm{Y}$ )

if and only if $\bar{R} X=\bar{R} Y$

$\mathrm{X}$ and $\mathrm{Y}$ are rough equal (X R_eq $\mathrm{Y}$ ) if and only if

$$
\text { (X b_R_eq Y) and (X t_R_eq Y). }
$$

\section{E. Other approximate equalities}

As noted earlier, the notion of rough equalities boils down to finding out equalities of sets in terms of the equalities of their lower and upper approximations. A much more generalised approximate equality which is almost independent of equality of sets, called the rough equivalence was introduced by Tripathy et al [13]. Later on two more types of approximate equalities called the approximate rough equality and approximate rough equivalence were introduced by Tripathy [15]. An interesting analysis on the effectiveness of these four types of approximate equalities have been carried out in [15], which shows that the notion of rough equivalence is the most general among the four; where as the notion of approximate rough equivalence is the most natural one from the application point of view. Some algebraic properties of rough equivalence have been established in [16].

Novotny and Pawlak [3] established eleven properties of rough equalities and stated that the corresponding replacement properties obtained by interchanging the top and bottom rough equalities do not hold. However, it was shown by Tripathy et al [13] that some of the replacement properties hold true and others hold true under suitable sufficient conditions. Validity of similar properties has been verified for all the other three types of approximate equalities. Algebraic properties involving rough sets using approximate equalities have been considered in [16, 18]. As far as multigranular rough sets are concerned, the rough equality and the rough equivalence have been extended to define the corresponding approximate equalities so far [14, 17].

In this paper we focus on defining multigranular approximate rough equalities, check the validity of the eleven direct properties and the corresponding replacement properties. We take a real life database to verify some of the results and also to construct counter examples.

\section{MULTIGRANULAR ROUGH EQUALITIES}

The concepts of rough equalities for basic rough sets were extended to the setting of multigranular rough sets by Tripathy et al [14]. A lso, the concepts of multigranular rough equivalences have been introduced and their properties have been studied. We state below the different types of multigranular approximate equalities, which have been introduced so far.

Definition 4: Let $\mathrm{R}$ and $\mathrm{S}$ be two equivalence relations on $\mathrm{U}$ and $\mathrm{X}, Y \subseteq U$. Then

$\mathrm{X}$ and $\mathrm{Y}$ are pessimistic bottom multigranular rough equal to each other with respect to $R$ and $S$ (X $\mathrm{Pb} \_\mathrm{R} * \mathrm{~S} \_$eq Y) if and only if $\underline{R * S} X=\underline{R^{*} S Y}$. (16)

$\mathrm{X}$ and $\mathrm{Y}$ are pessimistic top multigranular rough equal to each other with respect to $\mathrm{R}$ and $\mathrm{S}$ (X Pt_R*S_eq Y)

$$
\text { if and only if } \overline{R * S} X=\overline{R * S} Y \text {. }
$$

$\mathrm{X}$ and $\mathrm{Y}$ are pessimistic multigranular rough equal to each other with respect to $R$ and $S$ ( $X P_{-} R^{*} S_{-}$eq $Y$ ) if and only if

$$
\underline{R * S} X=\underline{R * S} Y \text { and } \overline{R^{*} S} X=\overline{R^{*} S} Y
$$

Definition 5: Let $\mathrm{R}$ and $\mathrm{S}$ be two equivalence relations on $\mathrm{U}$ and $\mathrm{X}, Y \subseteq U$. Then

$\mathrm{X}$ and $\mathrm{Y}$ are optimistic bottom multigranular rough equal to each other with respect to $R$ and $S$ ( $X$ Ob_R+S_eq Y) if and only if

$$
\underline{R+S} X=\underline{R+S Y} .
$$

$\mathrm{X}$ and $\mathrm{Y}$ are optimistic top multigranular rough equal to each other with respect to $\mathrm{R}$ and $\mathrm{S}$ (X Ot_R+S_eq Y) if and only if

$$
\overline{R+S} X=\overline{R+S} Y
$$


$\mathrm{X}$ and $\mathrm{Y}$ are optimistic multigranular rough equal to each other with respect to $\mathrm{R}$ and $\mathrm{S}$ ( $\mathrm{X} \mathrm{O} \mathrm{O}_{-} \mathrm{R}+\mathrm{S} \mathrm{Y}$ ) if and only if

$$
\underline{R+S} X=\underline{R+S} Y \text { and } \overline{R+S} X=\overline{R+S} Y .
$$

The notions of multigranular rough equivalences were introduced by Tripathy et al in [17], which are as follows:

Definition 6: Let $\mathrm{R}$ and $\mathrm{S}$ be two equivalence relations on $\mathrm{U}$ and $\mathrm{X}, Y \subseteq U$. Then,

$\mathrm{X}$ and $\mathrm{Y}$ are pessimistic bottom multigranular rough equivalent to each other with respect to $R$ and $S$ (X Pb_R*S_eqv Y) if and only if

$$
\underline{R * S} X \text { and } \underline{R * S} Y \text { are } \phi \text { or not } \phi \text { together. }
$$

$\mathrm{X}$ and $\mathrm{Y}$ are pessimistic top multigranular rough equivalent to each other with respect to $\mathrm{R}$ and $\mathrm{S}$ (X Pt_R*S_eqv Y) if and only if $\overline{R * S} X$ and $\overline{R * S} Y$ are equal to $\mathrm{U}$ or not $\mathrm{U}$ together.

$\mathrm{X}$ and $\mathrm{Y}$ are pessimistic mu ltigranular rough equivalent to each other with respect to $\mathrm{R}$ and $\mathrm{S}$ ( $\mathrm{X} \mathrm{P}_{-} \mathrm{R} * \mathrm{~S}$ _eqv $\mathrm{Y}$ ) if and only if $R^{*} S X$ and $\underline{R^{*} S} Y$ are $\phi$ or not $\phi$ together and $\overline{R * S} X$ and $\overline{R * S} Y$ are equal to $\mathrm{U}$ or not $\mathrm{U}$ together.

Definition 7: Let $\mathrm{R}$ and $\mathrm{S}$ be two equivalence relations on $\mathrm{U}$ and $\mathrm{X}, \mathrm{Y} \subseteq \mathrm{U}$. Then,

$\mathrm{X}$ and $\mathrm{Y}$ are pessimistic bottom multigranular rough equivalent to each other with respect to $\mathrm{R}$ and $\mathrm{S}$ (X $\mathrm{Pb} \_\mathrm{R}+\mathrm{S} \_$eqv $\mathrm{Y}$ ) if and only if $\underline{R+S} X$ and $\underline{R+S Y}$ are $\phi$ or not $\phi$ together.

$\mathrm{X}$ and $\mathrm{Y}$ are pessimistic top multig ranular rough equivalent to each other with respect to $\mathrm{R}$ and $\mathrm{S}$ (X Pt_R+S_eqv Y) if and only if $\overline{R+S} X$ and $\overline{R+S} Y$ are equal to $\mathrm{U}$ or not $\mathrm{U}$ together.

$\mathrm{X}$ and $\mathrm{Y}$ are pessimistic multigranular rough equivalent to each other with respect to $\mathrm{R}$ and $\mathrm{S}$ ( $\mathrm{X} \mathrm{P} \_\mathrm{R}+\mathrm{S} \_$eqv $\mathrm{Y}$ ) if and only if $\underline{R+S} X$ and $\underline{R+S} Y$ are $\phi$ or not $\phi$ together and $\overline{R+S} X$ and $\overline{R+S} Y$ are equal to $\mathrm{U}$ or not $\mathrm{U}$ together.

\section{APPROXIMATE MULTIGRANULAR ROUGH EQUALITIES}

In this section we introduce the notions of approximate rough equalities and study their properties. First, we define the two types of approximate mu ltigranular rough equalities below.

Definition 8: Let $\mathrm{R}$ and $\mathrm{S}$ be two equivalence relations on $\mathrm{U}$ and $\mathrm{X}, Y \subseteq U$. Then

$\mathrm{X}$ and $\mathrm{Y}$ are pessimistic bottom multigranular approximate rough equal with respect to $R$ and $S$ (X $\mathrm{Pb} \_\mathrm{R} * \mathrm{~S}$ aeq $\mathrm{Y}$ ) if and only if $\underline{R} * S X=R^{*} S Y$.
$\mathrm{X}$ and $\mathrm{Y}$ are pessimistic top multigranular appro ximate rough equal with respect to $\mathrm{R}$ and $\mathrm{S}$ (X Pt_R*S aeq $\mathrm{Y}$ ) if and only if $R^{*} S X$ and $\underline{R} * S Y$ are equal to $\mathrm{U}$ or not $\mathrm{U}$ together.

$\mathrm{X}$ and $\mathrm{Y}$ are pessimistic multigranular approximate rough equal to each other with respect to $\mathrm{R}$ and $\mathrm{S}$ ( $\mathrm{X}$ P_R*S_eq Y) if and only if $R * S X=R^{*} S Y$ and $\underline{R * S} X$ and $\underline{R * S Y}$ are equal to $\mathrm{U}$ or not $\mathrm{U}$ together.

Definition 9: Let $\mathrm{R}$ and $\mathrm{S}$ be two equivalence relations on $\mathrm{U}$ and $\mathrm{X}, Y \subseteq U$. Then

$\mathrm{X}$ and $\mathrm{Y}$ are optimistic bottom multigranular approximate rough equal with respect to $R$ and $S$ (X $\mathrm{Pb} \_\mathrm{R}+\mathrm{S}$ aeq $\mathrm{Y}$ ) if and only if

$$
\underline{R+S} X=\underline{R+S Y} \text {. }
$$

$\mathrm{X}$ and $\mathrm{Y}$ are optimistic top mu ltigranular approximate rough equal with respect to $\mathrm{R}$ and $\mathrm{S}$ (X Pt_R+S aeq $\mathrm{Y}$ ) if and only if $\overline{R+S} X$ and $\overline{R+S} Y$ are equal to $\mathrm{U}$ or not $\mathrm{U}$ together.

$\mathrm{X}$ and $\mathrm{Y}$ are optimistic multigranular approximate rough equal to each other with respect to $\mathrm{R}$ and $\mathrm{S}$ ( $\mathrm{X}$ P_R+S_eq Y) if and only if $\underline{R+S} X=\underline{R+S Y}$ and $\overline{R+S} X$ and $\overline{R+S} Y$ are equal to $\mathrm{U}$ or not $\mathrm{U}$ together.

\section{A. Properties of Optimistic Multigranular Approximate Rough Equalities}

In this section, we shall deal with the properties of optimistic multigranular approximate equalities of rough sets. First, we establish some basic properties in the next subsection.

\section{B. Basic properties}

Property 1: $\mathrm{X} \quad \mathrm{Ob} \_\mathrm{R}+\mathrm{S} \_$aeq $\quad \mathrm{Y}$ if $X \cap Y$ Ob_R+S_aeq $\mathrm{Y}$ and $X \bigcap Y$ Ob_R+S_aeq $\mathrm{Y}$. The converse may not be true

Proof: The if part is obvious by definition. Next, suppose X Ob_R+S_eq Y. Then by definition $\underline{R+S} X=\underline{R+S Y}$.

But by (2.2.5) all that we can conclude is that $\quad \underline{R+S}(X \cap Y) \subseteq \underline{R+S}(X) \quad$ and $\underline{R+S}(X \cap Y) \subseteq \underline{R+S}(\mathrm{Y})$. We may not get the equality in either case.

Property 2: $X$ Ot_R+S_aeq $Y$ if $X \bigcup Y$ Ot_R+S_aeq $\mathrm{Y}$ and $\mathrm{X} \cup \mathrm{Y}$ Ot_R+S_aeq $\mathrm{Y}$. The converse may not be true

Proof: The first part is clear by definition. However by (8) the converse may fail to be true when both $\overline{R+S} X$ and $\overline{R+S Y}$ are not $\mathrm{U}$ but $\overline{R+S}(\mathrm{X} \cup \mathrm{Y})$ is equal to $\mathrm{U}$. 
Property 3: $X$ Ot_R+S_aeq $X^{\prime}$ and $Y$ Ot_R+S_aeq $Y^{\prime}$ may not imply that $\bar{X} \cup \bar{Y}$ Ot_R+S_aeq $\mathrm{X}^{\prime} \cup \mathrm{Y}^{\prime}$

Proof: Follows from (8) and the case when $\overline{R+S}(\mathrm{X}), \overline{R+S}\left(X^{\prime}\right), \overline{R+S}(\mathrm{Y}), \overline{R+S}\left(Y^{\prime}\right)$ are all not equal to $\mathrm{U}$ where as one of $\overline{R+S}(\mathrm{X} \cup \mathrm{Y})$ and $\overline{R+S}\left(\mathrm{X}^{\prime} \cup \mathrm{Y}^{\prime}\right)$ is not equal to $\mathrm{U}$ where as the other one is equal to $U$.

Property 4: $\mathrm{X} O \mathrm{Ob}_{-} \mathrm{R}+\mathrm{S} \_$aeq $\mathrm{X}^{\prime}$ and $\mathrm{Y} \mathrm{Ob} \_\mathrm{R}+\mathrm{S} \_$aeq $\mathrm{Y}^{\prime}$ may not imply that $X \cap Y$ Ob_R+S_aeq $\mathrm{X}^{\prime} \cap \mathrm{Y}^{\prime}$.

Proof: Follows from (5).

Property 5: If $\mathrm{X}$ Ot_R+S_aeq $\mathrm{Y}$ then $\mathrm{XU}-\mathrm{Y}$ Ot_R+S_aeq U.

Proof: Follows from (8). and as $\overline{R+S}(X \cup-Y) \supseteq \overline{R+S}(X) \cup \overline{R+S}(-Y)$

$\supseteq \overline{R+S}(X) \cup-(\underline{R+S}(Y))=\mathrm{U}$.

Property 6: If $\mathrm{X} \quad \mathrm{Ob} \_\mathrm{R}+\mathrm{S} \_$aeq $\mathrm{Y}$ then $\mathrm{X} \cap-\mathrm{Y}$ Ob_R+S_aeq $\phi$.

Proof: Follows from (5) and as $\underline{R+S}(X \cap-Y) \subseteq \underline{R+S}(X) \bigcap \underline{R+S}(-Y)$ $\subseteq \underline{R+S}(X) \bigcap-(\overline{R+S}(Y))=\underline{R+S}(\mathrm{Y}) \bigcap-(\overline{R+S}(Y))=\phi$.

Property 7: If $\mathrm{X} \subseteq \mathrm{Y}$ and $\mathrm{Y}$ Ot_R+S_aeq $\phi$ then $\mathrm{X}$ Ot_R+S_aeq $\phi$

Proof: $\mathrm{As} \mathrm{X} \subseteq \mathrm{Y}$, we have $\overline{R+S} \mathrm{X} \subseteq \overline{R+S} \mathrm{Y}$ and so if $\overline{R+S} Y=\phi$ then $\overline{R+S} X=\phi$

Property 8: If $\mathrm{X} \subseteq \mathrm{Y}$ and $\mathrm{X}$ Ot_R+S_aeq $\mathrm{U}$ then $\mathrm{Y}$ Ot_R+S_aeq U.

Proof: $\quad \frac{\mathrm{As}}{R+S} \mathrm{X} \subseteq \overline{R+S} \mathrm{Y}$ and so if $\frac{,}{R+S \lambda=U}$ then
$\overline{R+S Y}=U$.

Property 9: If $X$ Ot_R+S_aeq $Y$ then it may not be true that $-\mathrm{X}$ Ob_R+S_aeq $-\mathrm{Y}$.

Proof: The property fails to be true when $\overline{R+S}(X)$ and $\overline{R+S}(\mathrm{Y})$ are not equal to $\mathrm{U}$ and unequal.

Property 10: If $\mathrm{X}$ Ob_R+S_aeq $\phi$ or $\mathrm{Y} O b \_\mathrm{R}+\mathrm{S} \_$aeq $\phi$ then $X \cap Y$ Ob_R+S_aeq $\phi$.

Proof: It follows from (5).

Property 11: If $X$ Ot_R+S_aeq $U$ or $Y$ Ot_R+S_aeq U then XUY Ot_R+S_aeq U.

Proof: It follows from (8) and definition of optimistic top multigranular approximate rough equality.

\section{Replacement properties}

In this subsection, we shall deal with the replacement properties of optimistic multigranular approximate equalities of rough sets.
Property 12: $\mathrm{X} \quad \mathrm{Ot} \_\mathrm{R}+\mathrm{S} \_$aeq $\quad \mathrm{Y}$ if $X \cap Y$ Ot_R+S_aeq $\mathrm{Y}$ and $X \cap Y$ Ot_R+S_aeq $\mathrm{Y}$. The converse may not be true.

Proof: The proof of if part follows directly from definition of optimistic top multigranular approximate rough equality.

From (7) the other part may not be true.

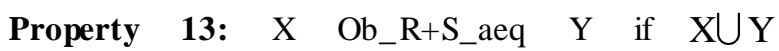
Ob_R+S_aeq $Y$ and $X \cup Y$ Ob_R+S_aeq $Y$. The converse may not be true.

Proof: the if part follows from definition. From (6) the other part may not be true.

Property 14: $X$ Ob_R+S_aeq $X{ }^{\prime}$ and $Y O b \_R+S \_$aeq $Y^{\prime}$ may not imply that $X \bigcup \bar{Y}$ Ob_R+S_aeq $X^{\prime} \bigcup Y^{\prime}$.

Proof: We provide an example to establish the claim. Let us take the information table 1.

$\mathrm{U}=\{$ Sam, Ram, Shyam... Pretha $\}$

U/Division $=\{\{$ Sam, Smith, Jacob $\},\{$ Shyam, John, Keny, lakman, Pretha\}, \{Peter, Albert, Linz, Sita , \{Roger, Mishra, Williams, Fatima $\},\{$ Ram, Hari, Martin, Biswas \}\}

$\mathrm{U} /$ Grade $=\{\{$ Shyam, Albert, Mishra, Martin, Jacob $\}$, \{Sam, John, Sita, Fatima $\}$, \{Peter, Smith, Williams, Lakman \}, \{Ram, Roger, Keny $\},\{$ Linz, Biswas, Pretha, Hari\}\}

U/Top Degree $=\{\{$ Shyam, Albert, Mishra, Martin, Jacob \}, \{Sam John $\}$, \{Sita, Fatima $\},\{$ Ram, Peter, Roger, Hari, Smith, Keny, Linz, Williams, Lakman, Biswas, Pretha\}\}

Let Division $=\mathrm{R}$ and Grade $=\mathrm{S}$,

$\mathrm{X}=\{$ Sam, Bis was, Keny, Smith, Jacob $\}$

$X^{\prime}=\{$ Hari, Sam, John, Smith, Jacob $\}$

$\mathrm{Y}=\{$ Roger, Peter, Fatima, Ram, Keny $\}$

$Y^{\prime}=\{$ Ram, Jacob, Sita, Keny, Fatima $\}$

$X \cup Y=\{$ Sam, Biswas, Keny, Smith, Jacob, Roger,

Peter, Fatima, Ram\}

$X^{\prime} \cup Y^{\prime}=\{$ Hari, Sam, John, Smith, Fatima, Ram, Jacob, Sita, Keny $\}$

$\underline{\mathrm{R}+\mathrm{S} X}=\underline{\mathrm{R}+\mathrm{S}} \mathrm{X}^{\prime}=\{$ Sam, Smith, Jacob $\}$

$\underline{\mathrm{R}+\mathrm{S}} \mathrm{Y}=\underline{\mathrm{R}+\mathrm{S}} \mathrm{Y}^{\prime}=\{$ Ram, Roger, Keny $\}$

$\underline{\mathrm{R}+\mathrm{S}}(\mathrm{XUY})=\{$ Sam, Smith, Jacob, Ram, Roger, Keny $\}$

$\underline{\mathrm{R}+\mathrm{S}}\left(X^{\prime} U^{\prime}\right)=\{$ Sam, Smith, Jacob, Ram, Roger,

Keny, Sam, Fatima, Sita, John $\}$

Hence $\underline{R+S}(X \cup Y) \neq \underline{R+S}\left(X^{\prime} \cup Y^{\prime}\right)$.

Property 15: $X$ Ot_R $+\mathrm{S} \_$aeq $\mathrm{X}^{\prime}$ and $\mathrm{Y}$ Ot_R+S_aeq $\mathrm{Y}^{\prime}$ may not imply that $X \cap \bar{Y}$ Ot_R+S_aeq $\mathrm{X}^{\prime} \cap \overline{\mathrm{Y}^{\prime}}$.

Proof: The property fails to be true when

$\overline{R+S}(\mathrm{X}), \overline{R+S}\left(X^{\prime}\right), \overline{R+S}(\mathrm{Y}), \overline{R+S}\left(Y^{\prime}\right) \quad$ are $\quad$ all equal to $\mathrm{U}$ but out of $\overline{R+S}(\mathrm{X} \cap \mathrm{Y})$ and $\overline{R+S}\left(\mathrm{X}^{\prime} \cap \mathrm{Y}^{\prime}\right)$ one is equal to $\mathrm{U}$ but the other one is not.

$X=\{$ Sam, Shyam, Peter, Martin, Fatima $\}$

$\mathrm{Y}=\{$ Shyam, Sam, Peter, Ram, Linz $\}$

$(X \cap Y)=\{$ Shyam, Sam, Peter $\}$

$X^{\prime}=\{$ Smith, Ram, Pretha, Roger, Sita $\}$

$Y^{\prime}=\{$ Keny, Albert, Sita, Bis was, Williams $\}$ 


$$
\left(X^{\prime} \cap Y^{\prime}\right)=\{\text { Sita }\}
$$

$\overline{R+S} X=\overline{R+S} X^{\prime}=\{$ Sam, Jacob, Smith, Shyam, John, Keny, Lakman, Pretha, Peter, Albert, Linz, Sita, Roger, Mishra, Williams, Fatima, Biswas, Pretha, Hari, Martin, Ram\}

$\overline{R+S} Y=\overline{R+S} Y^{\prime}=\{$ Sam, Jacob, Smith, Shyam, John, Keny, Lakman, Pretha, Peter, Albert, Linz, Sita, Roger, Mishra, Williams, Fatima, Biswas, Pretha, Hari, Martin, Ram\}
$\overline{R+S}(X \cap Y)=\{$ Shyam, John, Keny, Lakman, Pretha, Albert, Mishra, Martin, Sam, Smith, Jacob, John, Sita, Fatima, Peter, Albert, Linz, Williams \}

$\overline{R+S}\left(X^{\prime} \cap Y^{\prime}\right)=\{$ Peter, Albert, Linz, Sita, Sam, John, Fatima

Table 1. Faculty database

\begin{tabular}{|c|l|l|l|l|}
\hline S.N. & \multicolumn{1}{|c|}{ Name } & \multicolumn{1}{|c|}{ Division } & \multicolumn{1}{c|}{ Top Degree } \\
\hline 1. & Sam & Network & Assistant professor & MCA \\
\hline 2. & Ram & Information system & Professor & Ph.D \\
\hline 3. & Shyam & Software Engineering & Assistant professor (junior) & M.Sc. \\
\hline 4. & Peter & Artificial Intelligence & Associate Professor & Ph.D \\
\hline 5. & Roger & Embedded system & Professor & Ph.D \\
\hline 6. & Albert & Artificial Intelligence & Assistant Professor(Junior) & M.Sc \\
\hline 7. & Mishra & Embedded System & Assistant Professor(junior) & M.Sc. \\
\hline 8. & Hari & Information systems & Senior Professor & Ph.D \\
\hline 9. & John & Software Engineering & Assistant professor & MCA \\
\hline 10. & Smith & Network & Associate professor & Ph.D \\
\hline 11. & Linz & Artificial Intelligence & Senior Professor & Ph.D \\
\hline 12. & Keny & Software Engineering & Professor & Ph.D \\
\hline 13. & Williams & Embedded Systems & Associate Professor & Ph.D \\
\hline 14. & Martin & Information Systems & Assistant professor (junior) & M.Sc. \\
\hline 15. & Jacob & Network & Assistant professor (junior) & M.Sc. \\
\hline 16. & Lakman & Software Engineering & Associate Professor & Ph.D \\
\hline 17. & Sita & Artificial Intelligence & Assistant Professor & Ph.D \\
\hline 18. & Fatima & Embedded Systems & Assistant Professor & M.Tech \\
\hline 19. & Biswas & Information Systems & Senior Professor & M.Tech \\
\hline 20. & Pretha & Software Engineering & Senior Professor & Ph.D \\
\hline
\end{tabular}

Property 16: If $X \mathrm{Ob} \_\mathrm{R}+\mathrm{S} \_$aeq $\mathrm{Y}$ then it may not be true that $X \cup-Y$ Ob_R+S_aeq U.

Proof: We refer to table 1. Suppose,

$\mathrm{X}=\{$ Sam, Smith, Keny, Jacob $\} \mathrm{Y}=\{$ Roger, John, Peter, Smith, Jacob $\}$

$X \bigcup-Y=\{$ Sam, Keny, Ram, Shyam, Albert, Mishra, John, Hari, Smith, Linz, Keny, Williams, Martin, Lakman, Sita, Fatima, Biswas, Pretha

$\underline{\mathrm{R}+\mathrm{S}} X=\underline{\mathrm{R}+\mathrm{S}} \mathrm{Y}=\{$ Sam, Smith, Jacob $\}$

$\underline{\mathrm{R}+\mathrm{S}} X \cup-Y=\{$ Ram, Hari, Martin, Biswas, Linz, Pretha, Keny, Lakman, Sam, John, Sita, Fatima

Property 17: If $X$ Ot_R+S_aeq $Y$ then it may not be true that $X \cap-Y$ Ot_R+S_aeq $\phi$.

Proof: Follows from (7).

Property 18: If $X \subseteq Y$ and $\mathrm{Y} O \mathrm{Ob}_{-} \mathrm{R}+\mathrm{S} \_$aeq $\phi$ then $\mathrm{X}$ Ob_R+S_aeq $\phi$.
Proof: As $\mathrm{X} \subseteq \mathrm{Y}$, we have $\underline{R+S}(X) \subseteq R+S(\mathrm{Y})$. So,

The claim is true.

Property 19: If $\mathrm{X} \subseteq \mathrm{Y}$ and $\mathrm{X} O \mathrm{Ob} \_\mathrm{R}+\mathrm{S} \_$aeq $U$ then $\mathrm{Y}$ Ob_R+S_aeq U.

Proof: Similar to property 18.

Property 20: If $\mathrm{X} \quad \mathrm{Ob} \_\mathrm{R}+\mathrm{S} \_$aeq $\mathrm{Y}$ then $-\mathrm{X}$ Ot_R+S_aeq - Y.

Proof: $\quad X \quad O b \_R+S \_a e q \quad Y$ $\Rightarrow \underline{R+S}(X)=\underline{R+S}(\mathrm{Y})$. So,

$\overline{R+S}(-X)=-\underline{R+S}(X)=-\underline{R+S}(\mathrm{Y})=\overline{R+S}(-Y)$

. Hence the proof follows.

Property 21: If $X$ Ot_R $+S \_$aeq $\phi$ or $Y$ Ot_R+S_aeq $\phi$ then $X \cap Y$ Ot_R+S_aeq $\phi$.

Proof: Directly follows from (7). 
Property 22: If $\mathrm{X}$ Ob_R+S_aeq $\mathrm{U}$ or $\mathrm{YOb} \mathrm{Ob} \mathrm{R}+\mathrm{S} \_$aeq $\mathrm{U}$ then $X \cup Y$ Ob_R+S_aeq $\mathrm{U}$.

Proof: The proof follows from (6).

\section{Properties of Pessimistic Multigranular Approximate Rough Equalities}

In this section we establish properties of pessimistic multigranular appro ximate equalities of rough sets. First, we shall consider some general properties of optimistic multigranular rough sets in the next subsection. It is worth noting that the properties (9) to (12) are similar in nature to that in the single granulation case. These properties are to be used in establishing the properties of pessimistic multigranular approximate rough equalities.

We define the following concepts which are to be used in establishing these properties. It is worth noting that some of the properties in section A above can hold true if some additional conditions are imposed upon using these concepts as it was done in the single granulation case.

Definition 10: Let $\mathrm{R}$ and $\mathrm{S}$ be two equivalence relations on $\mathrm{U}$ and $\mathrm{X}, Y \subseteq U$. Then

We say that $X$ is optimistic bottom $\mathrm{R}+\mathrm{S}$ included in $Y$ iff $\underline{R+S} X \subseteq \underline{R+S Y}$.

We say that $\mathrm{X}$ is optimistic top $\mathrm{R}+\mathrm{S}$-included in $\mathrm{Y}$ iff $\overline{R+S} X \subseteq \overline{R+S} Y$.

We say that $\mathrm{X}$ is optimistic $\mathrm{R}+\mathrm{S}$-include in $\mathrm{Y}$ iff $\mathrm{X}$ is optimistic bottom $\mathrm{R}+\mathrm{S}$-included in $\mathrm{Y}$ and $\mathrm{X}$ is optimistic top $\mathrm{R}+\mathrm{S}$-included in $\mathrm{Y}$.

Definition 11: Let $\mathrm{R}$ and $\mathrm{S}$ be two equivalence relations on $\mathrm{U}$ and $\mathrm{X}, Y \subseteq U$. Then

We say that $X$ is pessimistic bottom $R^{*} \mathrm{~S}$ included in $Y$ iff $\underline{R * S} X \subseteq \underline{R * S Y}$.

We say that $\mathrm{X}$ is pessimistic top $\mathrm{R} * \mathrm{~S}$-included in $\mathrm{Y}$ iff $\overline{R * S} X \subseteq \overline{R * S} Y$.

We say that $\mathrm{X}$ is pessimistic $\mathrm{R}^{*} \mathrm{~S}$-included in $\mathrm{Y}$ iff $\mathrm{X}$ is optimistic bottom $\mathrm{R}^{*} \mathrm{~S}$-included in $\mathrm{Y}$ and $\mathrm{X}$ is pessimistic top $\mathrm{R}^{*} \mathrm{~S}$-included in $\mathrm{Y}$.

Definition 12: Let $\mathrm{R}$ and $\mathrm{S}$ be two equivalence relations on $\mathrm{U}$ and $\mathrm{X}, Y \subseteq U$. Then we say that

$\mathrm{X}$ and $\mathrm{Y}$ are optimistic $\mathrm{R}+\mathrm{S}$ bottom comparable iff $\mathrm{X}$ is optimistic bottom $\mathrm{R}+\mathrm{S}$-included in $\mathrm{Y}$ or $\mathrm{Y}$ is optimistic bottom $\mathrm{R}+\mathrm{S}$-included in $\mathrm{X}$ holds.

$\mathrm{X}$ and $\mathrm{Y}$ are optimistic $\mathrm{R}+\mathrm{S}$ top comparable iff $\mathrm{X}$ is optimistic top $\mathrm{R}+\mathrm{S}$-included in $\mathrm{Y}$ or $\mathrm{Y}$ is optimistic top $\mathrm{R}+\mathrm{S}$-included in $\mathrm{X}$ holds

$\mathrm{X}$ and $\mathrm{Y}$ are optimistic $\mathrm{R}+\mathrm{S}$ comparable iff $\mathrm{X}$ and $\mathrm{Y}$ are optimistic $\mathrm{R}+\mathrm{S}$ bottom comparable and $\mathrm{X}$ and $\mathrm{Y}$ are optimistic R+S top comparable.

Definition 13: Let $\mathrm{R}$ and $\mathrm{S}$ be two equivalence relations on $\mathrm{U}$ and $\mathrm{X}, Y \subseteq U$. Then we say that
$\mathrm{X}$ and $\mathrm{Y}$ are pessimistic $\mathrm{R}^{*} \mathrm{~S}$ bottom comparable iff $\mathrm{X}$ is pessimistic bottom $\mathrm{R}^{*} \mathrm{~S}$-included in $\mathrm{Y}$ or $\mathrm{Y}$ is pessimistic bottom $R^{*} S$-included in $\mathrm{X}$ holds.

$\mathrm{X}$ and $\mathrm{Y}$ are pessimistic $\mathrm{R}^{*} \mathrm{~S}$ top comparable iff $\mathrm{X}$ is pessimistic top $\mathrm{R}^{*} \mathrm{~S}$-included in $\mathrm{Y}$ or $\mathrm{Y}$ is pessimistic top $\mathrm{R} * \mathrm{~S}$-included in $\mathrm{X}$ holds

$\mathrm{X}$ and $\mathrm{Y}$ are pessimistic $\mathrm{R}+\mathrm{S}$ comparable iff $\mathrm{X}$ and $\mathrm{Y}$ are pessimistic $\mathrm{R}+\mathrm{S}$ bottom comparable and $\mathrm{X}$ and $\mathrm{Y}$ are pessimistic $\mathrm{R}+\mathrm{S}$ top comparable.

\section{ROUGH EQUALITIES BASED APPROXIMATE REASONING}

As mentioned by Zadeh, approximate reasoning is viewed as a process of approximate solution of a system of relational assignment equations. We can consider the approximate equalities in this sense providing approximate reasoning. The usual practice is to generalize the modus ponens used in discrete mathematics for generation of rules. But here, we have used it in the first sense when we mention approximate reasoning.

\section{CONCLUSION}

Rough equalities take human knowledge into account while dealing with equalities of sets. Four types of such equalities have been introduced in the literature. Extending the basic rough sets, which depend upon a single equivalence relation at a time, multigranular rough sets have been introduced recently. Combining these two concepts together, the multigranular rough equalities and multigranular rough equivalences were introduced and studied by Tripathy et al very recently. In this paper, we introduced and studied the third type such combinations, called the multigranular approximate rough equalities. Several properties of such equalities are derived.

\section{REFERENCES}

[1] Zadeh, L.A.: Fuzzy Sets, Information and control, vol.8, no.3, (1965), pp.338-353

[2] Pawlak, Z: Rough Sets, International Journal of Information and Computer Science, (1982), pp.341-346.

[3] Pawlak, Z: Rough Sets, Theoretical Aspects of Reasoning about Data, Kluwer Academic Publishers, (1991).

[4] Qian, Y.H and Liang, J.Y.: Rough set method based on Multi-granulations, Proceedings of the 5th IEEE Conference on Cognitive Informatics, vol.1, (2006),pp.297-304.

[5] Qian, Y.H., Liang, J.Y and Dang, C.Y.: Pessimistic rough decision, in: Proceedings of RST 2010, Zhoushan, China, (2010), pp. 440-449.

[6] Tripathy, B.K. and Nagaraju, M.: A Comparative Analy sis of Multigranular Approaches and on Topological Properties of Incomplete Pessimistic Multigranular Rough Fuzzy Sets, International Journal of Intelligent Systems and Applications, vol.11, (2012), pp.99-109. 
[7] Tripathy, B.K. and Raghavan, R.: Some Algebraic properties of Multigranulations and an Analysis of Multigranular Approximations of Classifications, International Journal of Information Technology and Computer Science,vol.7, (2013),pp.63-70.

[8] Tripathy, B.K. and Raghavan, R.: On Some Comparison Properties of Rough Sets Based on Multigranulations and Types of Multigranular Approximations of Classifications, I.J. Intelligent Systems and Applications, (2013), 06, pp.70-77.

[9] Novotny, M. and Pawlak, Z.: Characterization of Rough Top equalities and Rough Bottom Equalities", Bull. Polish Acad. Sci. Math., 33, (1985), pp.91-97.

[10] Novotny, M. and Pawlak, Z.: On Rough Equalities, Bull. Polish Acad. Sci. Math., 33, (1985), pp.99-104.

[11] Novotny, M. and Pawlak, Z.: Black Box Analysis and Rough Top Equality”, Bull. Polish Acad. Sci. Math., 33, (1985), pp.105-113.

[12] Tripathy, B.K.: On Approximation of classifications, rough equalities and rough equivalences, Studies in Computational Intelligence, vol.174, Rough Set Theory: A True Landmark in Data Analysis, Springer Verlag, (2009), pp.85- 136

[13] Tripathy, B.K., Mitra, A. and Ojha, J.: On Rough Equalities and Rough Equivalences of Sets, SCTC 2008Akron, U.S.A., Springer-Verlag B erlin Heidelberg (2008), LNAI 5306, pp.92-102.

[14] Tripathy, B.K. and Mitra, A.: On the approximate equalities of multigranular rough sets and approximate reasoning, Proceedings, $4^{\text {th }}$ IEEE International Conference on Computing, Communication and Networking Technologies (ICCCNT 2013), 4-6 July (2013).

[15] Tripathy, B.K. :An Analysis of Approximate Equalities based on Rough Set Theory, International Journal of Advanced Science and Technology vol. 31, June, (2011), pp.23-36.

[16] Tripathy, B.K., Mitra, A. and Ojha, J.: Rough Equivalence and Algebraic properties of Rough Sets, International Journal of Artificial Intelligence and Soft Computing, (Switzerland), vol.1, nos.2/3/4, (2009), pp. $271-289$.

[17] Tripathy, B.K. and Mitra, A.: On Approximate Equivalences of Multigranular Rough Sets and Approximate Reasoning, International Journal of Information Technology and Computer Science, 10, (2013), pp.103-113

[18] Tripathy, B.K. and Mitra, A.: Algebric Properties of Rough Sets using topological characterisations and Approximate Equalities, IEEE International Conference on Computational Intelligence and Computing Research, (2013), 978-1-4799-1597-2/13.

\section{Authors' Profiles}

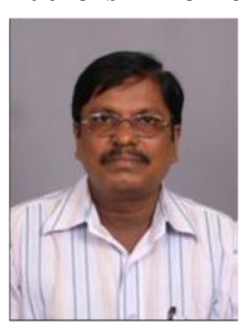

B.K.Tripathy is a senior professor in the school of computing sciences and engineering, VIT University, Vellore since 2007. He has produced $14 \mathrm{PhDs}, 13$ M.Phils and 01 M.S students so far. $\mathrm{He}$ has published more than 200 papers in different international journals, conference proceedings and edited research volumes. He has edited two research volumes for the IGI publications. He is in the editorial board or review panel of over 50 journals including Springer, Science Direct, IEEE and World Scientific publications. He is a life member/ senior member/member of 19 international forums including ACM,
IEEE, ACEEE and CSI. His current interest includes Fuzzy sets and systems, Rough sets and knowledge engineering, Multiset theory, List theory, Data clustering and database anony misation, content based learning, remote laboratories, Soft set analysis, Image processing ,cloud computing and Social Network Analy sis.

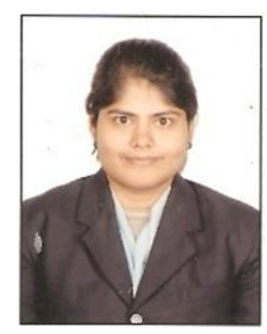

Rashmi Rawat received the degree in information technology engineering from RGPV University and at present she is pursuing her M.Tech degree from VIT University. Her research interests are rough sets, fuzzy sets, data clustering and data mining.

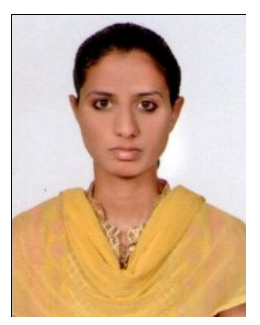

Divya Vani received her degree in computer science engineering from Andhra University in 2010 and is pursuing her M.Tech degree from VIT university. Her research interests are rough sets, Algorithm analysis and Data Mining.

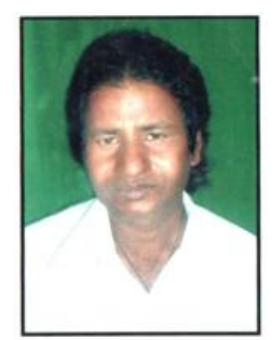

S.Ch.Parida is a lecturer in Mathematics in College, Odisha. He is pursuing his Ph.D under the supervision of Dr.B.K.Tripathy. He has a few papers published in international journals. His current research interest includes Rough sets and knowledge engineering and soft computing.

How to cite this paper: B. K. Tripathy, Rashmi Rawat, Divy a Vani .Y, Sudam Charan Parida,"Approximate Reasoning through Multigranular Approximate Rough Equalities", International Journal of Intelligent Systems and Applications(IJISA), vol.6, no.8, pp.69-76, 2014. DOI: 10.5815/ijisa.2014.08.08 